\title{
Carrier Phase Based Navigation Algorithm Design Using Carrier Phase Statistics in the Weak Signal Environment
}

\author{
Sul Gee Park ${ }^{1 \dagger}$, Deuk Jae Cho', Chansik Park ${ }^{2}$ \\ ${ }^{1}$ Maritime Safety Research Division, Korea Institute of Ocean Science and Technology, Daejeon 305-343, Korea \\ ${ }^{2}$ Department of Control and Robotics, Research Institute of Computer, Information and Communication, Chungbuk National \\ University, Cheongju 361-763, Korea
}

\begin{abstract}
Due to inaccurate safe navigation estimates, maritime accidents have been occurring consistently. In order to solve this, the precise positioning technology using carrier phase information is used, but due to high buildings near inland waterways or inclination, satellite signals might become weak or blocked for some time. Under this weak signal environment for some time, the GPS raw measurements become less accurate so that it is difficult to search and maintain the integer ambiguity of carrier phase. In this paper, a method to generate code and carrier phase measurements under this environment and maintain resilient navigation is proposed. In the weak signal environment, the position of the receiver is estimated using an inertial sensor, and with this information, the distance between the satellite and the receiver is calculated to generate code measurements using IGS product and model. And, the carrier phase measurements are generated based on the statistics for generating fractional phase. In order to verify the performance of the proposed method, the proposed method was compared for a fixed blocked time. It was confirmed that in case of a weak or blocked satellite signals for 1 to 5 minutes, the proposed method showed more improved results than the inertial navigation only, maintaining stable positioning accuracy within $1 \mathrm{~m}$.
\end{abstract}

Keywords: carrier phase based positioning, carrier phase statistics, weak signal environment

\section{INTRODUCTION}

Due to inaccurate safe navigation estimates, maritime accidents have been occurring consistently. In order to solve this, the precise positioning technology using carrier phase information is used, but due to tall buildings near inland waterways or inclination, satellite signals might become weak or blocked for some time. Currently, the air clearance of ship is determined by estimating approximately based on the draft of a ship when the ship passes through a marine bridge or facility such as suspension bridges and bridges connecting islands while sailing on the shore or inland waterways. Inaccurate estimates lead to secondary damages including huge restoration expenses for damages of bridges and power supply units as well as environmental pollution.

Received Aug 30, 2012 Revised Oct 10, 2012 Accepted Oct 23, 2012

†Corresponding Author

E-mail: parksg@kiost.ac

Tel: +82-42-866-3685 Fax: +82-42-866-3689
Therefore, it is necessary to determine the air clearance for safe navigation using the precise positioning technology.

Carrier phase measurements should be used together with code measurements for precise positioning. For the pseudorange measurements, the distance between the satellite and the receiver on the ground is predicted using the time delay of $\mathrm{C} / \mathrm{A}$ code, but for the prediction of distance by carrier phase measurements, changes in carrier phase generated by the satellite is used. Since the wave length of carrier is significantly shorter than the length of $\mathrm{C} / \mathrm{A}$ code, the distance measured using the carrier phase is more precise than the distance measured using codes. But, Integer ambiguity which is carrier phase wavelength value existing between the satellite and the receiver in the carrier phase measurements, and It is necessary to obtain this integer ambiguity to measure an accurate distance. It is important to obtain and maintain the integer ambiguity in the carrier phase based precise positioning (Parkinson \& Spilker 1996). But, a satellite signal may become weak or blocked for some time due to high buildings and mountain 
near inland waterways or inclination. And also, in case of a small ship, the satellite signal may be blocked near a large ship on the shore, and the satellite signal may also become weak or blocked in the ocean due to an oil or natural gas rig. In this weak signal environment for some time, the GPS measurements become less accurate so that it is difficult to search and maintain the integer ambiguity of carrier phase, and the satellite constellation becomes inadequate so that the positioning becomes less accurate. And also, due to frequent radio disturbances by North Korea, a problem arises regarding that small ships mainly depending on GPS (Global Positioning System) might drift or wreck by bad weather, and there is a rising interest on studies showing continuing and stable positioning results under a weak signal environment (Melgard et al. 2011).

An alternative sensor used most frequently when a satellite signal is weak or blocked is the inertial sensor. However, due to error increase as time goes on in the inertial sensor, there is a difficulty in satisfying RMS error within $1 \mathrm{~m}$ in order to estimate the air clearance of ship for safe navigation (Titterton \& Weston 2004). Calgary University in Canada uses GPS and GLONASS measurements together in case satellite signals are blocked in one direction in the ocean due to oil rigs and facility to improve the geometry of satellites slightly but in this case the time error related to the GLONASS should be also considered (Melgard et al. 2011). Sydney University in Australia uses image sensors and inertial sensors but there is the problem of expense due to the usage of additional sensors (Kim \& Sukkarieh).

In this paper, the algorithm to generate code measurements and carrier phase measurements of GPS using carrier phase statistics without an additional sensor except the inertial sensor is proposed. Chapter 2 explains the algorithm to generate code measurements, and Chapter 3 and 4 explain the algorithm to generate carrier phase measurements. Chapter 5 explains the configuration and result of performance verification and presents the result and future tasks lastly.

\section{GENERATION OF CODE MEASUREMENTS}

The carrier phase based navigation algorithm using carrier phase statistics and inertial sensor in the weak signal environment which is proposed in this paper is as shown in Fig. 1. In this paper, Signal to Noise Ratio of the satellite with elevation angle over more than $15^{\circ}$ is considered as a weak or blocked satellite signal when its value is under $20 \mathrm{~dB}$, and the code and carrier phase measurements of the weak or blocked satellite signal is generated using the proposed algorithm. It is possible to carry out continuing and stable navigation using the measurements generated with the proposed method.

At first, the code measurements $(P)$ is given by the Eq. (1) (Teunissen \& Kleusberg 1997).

$$
P=\rho+c(d t-d T)+T+I+\omega_{P}
$$

Here, $\rho$ is the distance between the satellite and the receiver, $c$ is the speed of light, $d t$ is the clock error related to the receiver, $d T$ is the clock error related to the satellite, $T$ is the tropospheric delay error, $I$ is the ionospheric delay error, and $\omega_{P}$ is the noise of the code measurements.

The first step to generate code measurements is generation of the distance between the satellite and the receiver $(\rho)$, the clock error related to the receiver and satellite, the tropospheric delay error and other errors as shown in Fig. 1. At first, the distance between the satellite and the receiver is calculated using the precise orbit of satellite provided by International GNSS Service (IGS) and the position of the receiver calculated from inertial sensor. At this time, the position of the receiver is calculated with the following Eq. (2).

$$
X(t)=X_{G P S}(t-\Delta t)+X_{I N S}(\Delta t)
$$

Here, $X$ is the position of the receiver, and the subscript is the sensor of which position is calculated.

The position of the receiver in current epoch $(t)$ is calculated by adding the position of the receiver $\left(X_{G P S}(t-\Delta t)\right)$ estimated with the carrier phase-based precise navigation in the previous epoch $((t-\Delta t))$ to the distance navigated for $(\Delta t)$ time $\left(X_{I N S}(\Delta t)\right)$ estimated using the inertial sensor as shown in the Eq. (2). Here, the position of the receiver in epoch $(t)$ is not for the navigation result for the current position of the receiver estimated using the inertial sensor, but for the generation of the distance between the satellite and the receiver. If the position of the receiver is calculated with navigation of the inertial sensor only under a weak signal environment for some time, error increases as time goes on, so that it is difficult to carry out precise navigation. However, error in the inertial navigation is not significant for a short period of time, the distance navigated for $\Delta t$ time is calculated with the inertial sensor to generate the measurements and precise current position of the receiver is calculated using the carrier phase based navigation proposed in this paper. It is inconvenient to follow a number of steps, but continuing and stable positioning results can be obtained.

Second, the clock error is estimated. Since the clock error 
related to the receiver is common error for all satellites in one receiver, and in case a satellite signal is weak or blocked partially, the time error related to the receiver estimated from other satellite signals is used, and in case all satellite signals are blocked, the clock error related to receiver for the previous epoch and its extrapolated values are used to calculate the clock error related to the receiver. Precise clock error related to the satellite provided by the IGS is used to calculate the clock error related to the satellite.

Third, the tropospheric delay error and other errors are calculated. The most widely used Saastamoinen model and Niell Mapping Function (NMF) are used to estimate the tropospheric delay error. The NMF is a model based on changes in the geological location rather than meteorological variables on the ground which is derived in the aspect of temperature and relative humidity, and the height and latitude changing on the mean surface of the sea were considered (Saastamoinen et al. 1972, Niell 1996). The ionospheric delay error and other errors also can be calculated through the model.

The sum total of the distance between the satellite and the receiver, clock errors, the atmospheric delay errors and other errors generated as above can generate code measurements as shown in Eq. (1). Here, it is assumed that there is no noise in code measurements, and in case of GPS only navigation, estimation of errors including tidal, phase wind-up and instrumental error is required, but these can be selected according to the method of carrier phase based precise navigation method or required performance.

\section{GENERATON OF CARRIER PHASE MEASUREMENTS}

Carrier phase measurements $(\Phi)$ is given by the Eq. (3) (Tiberius \& de Jonge 1995).

$$
\frac{\Phi}{\lambda}=\phi_{b}=\frac{1}{\lambda}(\rho+c(d t-d T)+T-I)+N+\phi_{f}+C_{R}+\omega_{\Phi}
$$

Here, $\phi_{b}$ is the carrier beat phase, $\lambda$ is the wavelength, $N$ is the integer ambiguity, $\phi_{f}$ is the fractional phase, and $C_{R}$ is the cycle ambiguity calculated from the previous time and current time.

In order to generate carrier phase measurements as shown in Fig. 1 and Eq. (3), the distance between the satellite and the receiver, the clock errors, the atmospheric delay errors and other errors as well as integer ambiguity, cycle ambiguity and fractional phase should be estimated. The carrier phase measurements have the characteristics

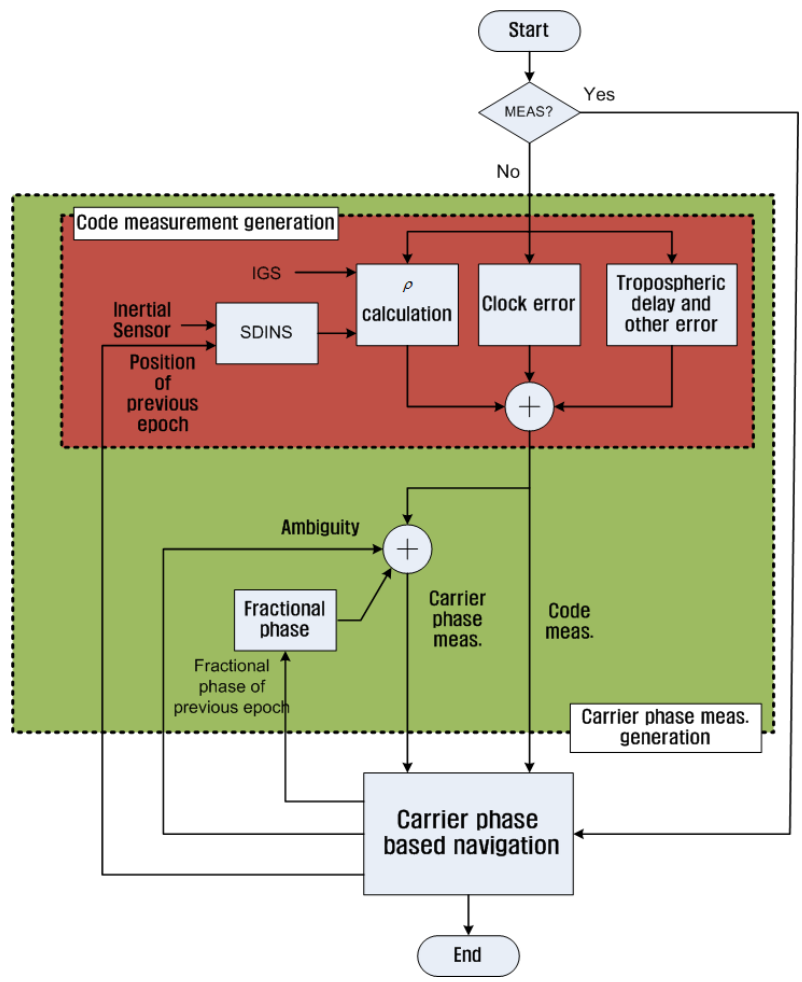

Fig. 1. Method of generating code and carrier phase measurements.

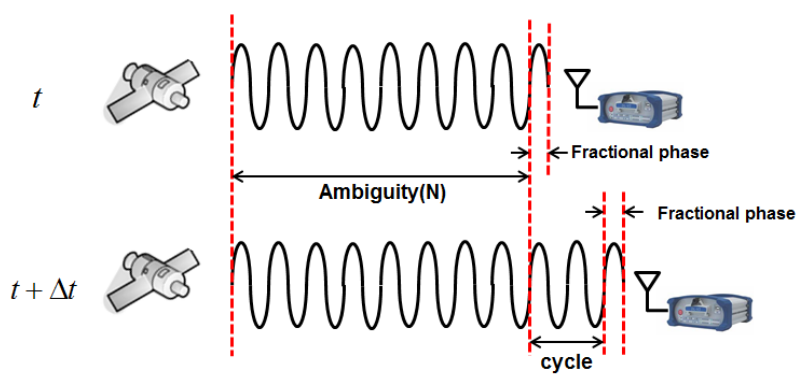

Fig. 2. Characteristics of carrier phase measurements.

as shown in Fig. 2. Here, one cycle is one wavelength of the carrier, and in case of L1 frequency signal, one cycle is approximately $0.19 \mathrm{~m}$, and in case of L2 frequency signal, one cycle is approximately $0.24 \mathrm{~m}$.

Since the integer ambiguity does not change as time goes on, carrier phase measurements can be estimated from changes in the cycle and fractional phase value. The cycle can be estimated from the pseudorange which changes as time goes on, but fractional phase values can be calculated only from actual measurements or accurate distance between the satellite and the receiver. In order to estimate the fractional phase value, a method of using statistics is proposed in this paper. 


\section{FRACTIONAL PHASE STATISTICS}

In order to obtain the fractional phase statistics, carrier phase measurements were received from the simulator. Spirent's GSS8000 was used for the GPS simulator, and NovAtel's DL-V3 was used for the receiver. In order to obtain more accurate fractional phase statistics, only tropospheric and ionospheric delay errors were applied to the simulator, and only L1 frequency measurements were generated without applying the orbit and the clock errors of the satellite. The simulation data in a $1 \mathrm{~Hz}$ interval for 3700 seconds in the static condition was acquired and generated into the statistics. The fractional phase was calculated with Eq. (3) as shown in Eq. (4).

$$
\phi_{f}=\frac{\Phi}{\lambda}-\frac{1}{\lambda}(\rho+c(d t-d T)+T-I)-N-C_{R}
$$

And then, 1 cycle $(0 \sim 2 \pi)$ was divided into 8 sections as shown in Fig. 3 and the statistics were obtained based on the number of fractional phases shown in each section for total 3700 seconds, and the result is shown in Table 1. As shown in Table 1, total number of fractional phases appeared in each section was similar in all 8 sections. This shows that

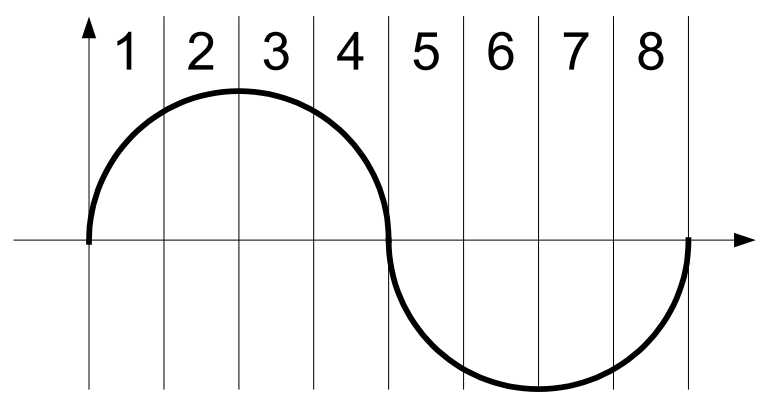

Fig. 3. Fractional phase of 1 cycle divided into 8 sections. the initial fractional phase is not maintained but it changes randomly within 1 cycle $(0 \sim 2 \pi)$.

However, when using fractional phase acquired at all sections in a similar frequency as shown in Table 1 and generating 8 sections for the fractional phase discretionally even though actual fractional phase section is 1 section, the error in the carrier phase measurements will be almost $0.19 \mathrm{~m}$ or $0.24 \mathrm{~m}$. Since this influences the result of precise positioning, it is necessary to determine fractional phases using more accurate statistics.

In order to confirm accurate fractional phase statistics and obtain the statistics for the relations between the fractional phase value of previous time and the fractional phase of current time, the carrier fractional phase statistics of 3 satellites including PRN 4, PRN 5 and PRN 12 in Table

Table 2. Sequential fractional phase statistics.

\begin{tabular}{|c|c|c|c|}
\hline \multicolumn{2}{|c|}{ Fractional phase sections } & \multirow{2}{*}{ Statistic } & \multirow{2}{*}{$\begin{array}{c}\text { Probability } \\
\text { (\%) }\end{array}$} \\
\hline Previous epoch & Current epoch & & \\
\hline \multirow[t]{3}{*}{1} & 8 & 337 & 24 \\
\hline & 1 & 524 & 37 \\
\hline & 2 & 347 & 25 \\
\hline \multirow[t]{3}{*}{2} & 1 & 346 & 26 \\
\hline & 2 & 509 & 38 \\
\hline & 3 & 318 & 24 \\
\hline \multirow[t]{3}{*}{3} & 2 & 308 & 23 \\
\hline & 3 & 523 & 39 \\
\hline & 4 & 353 & 26 \\
\hline \multirow[t]{3}{*}{4} & 3 & 330 & 23 \\
\hline & 4 & 549 & 38 \\
\hline & 5 & 361 & 25 \\
\hline \multirow[t]{3}{*}{5} & 4 & 344 & 25 \\
\hline & 5 & 503 & 37 \\
\hline & 6 & 312 & 23 \\
\hline \multirow[t]{3}{*}{6} & 5 & 314 & 23 \\
\hline & 6 & 512 & 38 \\
\hline & 7 & 327 & 24 \\
\hline \multirow[t]{3}{*}{7} & 6 & 322 & 23 \\
\hline & 7 & 548 & 39 \\
\hline & 8 & 337 & 24 \\
\hline \multirow[t]{3}{*}{8} & 7 & 318 & 23 \\
\hline & 8 & 517 & 37 \\
\hline & 1 & 358 & 26 \\
\hline
\end{tabular}

Table 1. Carrier fractional phase statistics of 8 satellites.

\begin{tabular}{|c|c|c|c|c|c|c|c|c|c|}
\hline & $\begin{array}{c}\text { Section } \\
\text { (Fractional } \\
\text { phase) }\end{array}$ & $\begin{array}{c}\text { PRN2 } \\
\text { (statistic) }\end{array}$ & $\begin{array}{c}\text { PRN4 } \\
\text { (statistic) }\end{array}$ & $\begin{array}{c}\text { PRN 5 } \\
\text { (statistic) }\end{array}$ & $\begin{array}{c}\text { PRN } 10 \\
\text { (statistic) }\end{array}$ & $\begin{array}{c}\text { PRN } 12 \\
\text { (statistic) }\end{array}$ & $\begin{array}{c}\text { PRN 24 } \\
\text { (statistic) }\end{array}$ & $\begin{array}{c}\text { PRN 26 } \\
\text { (statistic) }\end{array}$ & $\begin{array}{c}\text { PRN 29 } \\
\text { (statistic) }\end{array}$ \\
\hline \multirow{8}{*}{$\begin{array}{c}\text { Fractional } \\
\text { phase }\end{array}$} & $1\left(0 \sim \frac{2 \pi}{8}\right)$ & 422 & 476 & 478 & 508 & 464 & 400 & 551 & 459 \\
\hline & $2\left(\frac{2 \pi}{8} \sim \frac{4 \pi}{8}\right)$ & 499 & 497 & 441 & 482 & 413 & 440 & 519 & 583 \\
\hline & $3\left(\frac{4 \pi}{8} \sim \frac{6 \pi}{8}\right)$ & 498 & 444 & 465 & 439 & 445 & 550 & 416 & 605 \\
\hline & $4\left(\frac{6 \pi}{8} \sim \frac{8 \pi}{8}\right)$ & 507 & 476 & 492 & 391 & 474 & 548 & 346 & 546 \\
\hline & $5\left(\frac{8 \pi}{8} \sim \frac{10 \pi}{8}\right)$ & 517 & 422 & 467 & 429 & 463 & 556 & 370 & 401 \\
\hline & $6\left(\frac{10 \pi}{8} \sim \frac{12 \pi}{8}\right)$ & 448 & 421 & 450 & 446 & 470 & 456 & 411 & 315 \\
\hline & $7\left(\frac{12 \pi}{8} \sim \frac{14 \pi}{8}\right)$ & 390 & 445 & 457 & 487 & 489 & 375 & 523 & 322 \\
\hline & $8\left(\frac{14 \pi}{8} \sim \frac{16 \pi}{8}\right)$ & 398 & 498 & 429 & 497 & 461 & 354 & 543 & 448 \\
\hline \multicolumn{2}{|c|}{ Mean (\%) } & 12.5 & 12.5 & 12.5 & 12.5 & 12.5 & 12.5 & 12.5 & 12.5 \\
\hline \multicolumn{2}{|c|}{ Standard deviation } & 0.01316 & 0.00790 & 0.00513 & 0.0101 & 0.0057 & 0.02095 & 0.02106 & 0.02838 \\
\hline
\end{tabular}




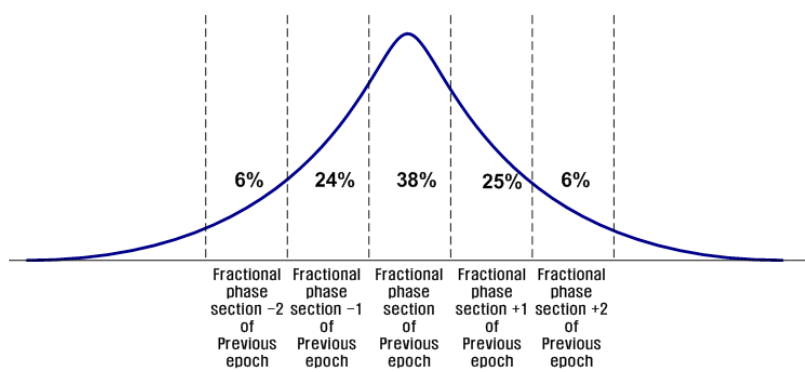

Fig. 4. Fractional phase statistics among current, previous, and future epochs.

1 were used. The number of statistics for PRN 4, PRN 5 and PRN 12 is distributed almost ideally with low standard deviation in comparison to the other satellites. The result of confirming fractional phase statistics acquired continuously from 3 satellites is as shown in Fig. 4 and Table 2.

The probability for the fractional phase of current epoch $(t)$ to be in the same section with the fractional phase of previous epoch $(t-\Delta t)$ is $38 \%$ in average, and probabilities for the fractional phase of current epoch $(t)$ to be in previous or next sections are $24 \%$ and $25 \%$ in average, and the probabilities for the fractional phase of current epoch $(t)$ to be in two sections before or after are $6 \%$ respectively. And the probability to be in the remaining sections is $1 \%$. If the fractional phase section of previous epoch $(t-\Delta t)$ before a satellite signal becomes weak or blocked is section 1 , the

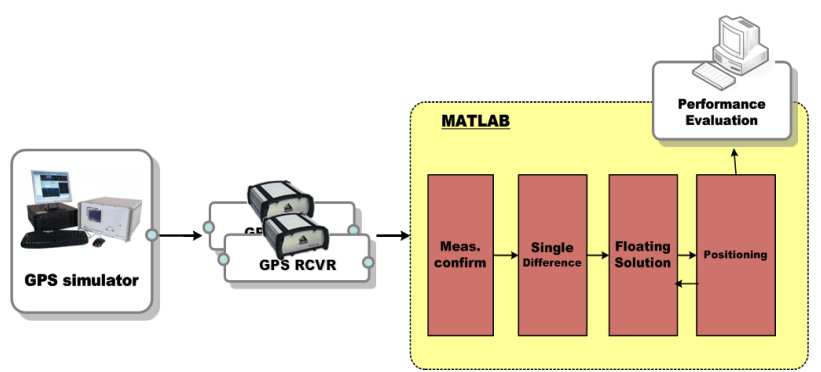

Fig. 5. Configuration of carrier phase based navigation algorithm in static condition. code measurements can be generated with the proposed method in Chapter 2 when the satellite signal becomes weak or blocked, and for determining the fractional phase from the carrier phase measurements, since the probability to be section 1 which is the same with previous fractional phase section is $37 \%$, the probabilities to be Sections 8 or 2 are $24 \%$ and $25 \%$ respectively, the probabilities to be Sections 7 or 3 are $6 \%$ respectively, and the probabilities to be Sections 4,5 and 6 are $0.3 \%$ respectively, it is possible to generate current fractional phase value based on these probabilities. If it is Section 2, the fractional phase value is $\frac{3 \pi}{8}\left(=\frac{3}{16} \cdot 2 \pi\right)$ which is the middle value of Section 2 in Table 1 , in case of L1 satellite signal, it is $\frac{3}{16} \cdot 0.19=0.03563(\mathrm{~m})$, and in case of L2 satellite signal, it is $\frac{3}{16} \cdot 0.24=0.045(\mathrm{~m})$, so it is possible to generate fractional phases. Currently, the whole section is divided into 8 sections for convenience, but according to the requirements of the precise positioning system, the fractional phase sections can be more subdivided.

Likewise, if the fractional phase sections of epoch $(t-\Delta t)$ are known, it is possible to estimate the fractional phase

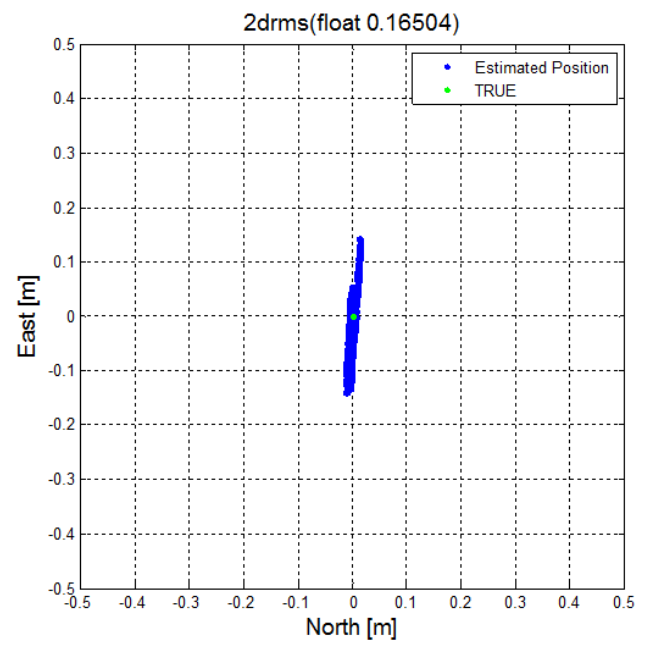

Fig. 6. The results of SDGPS test in static condition.

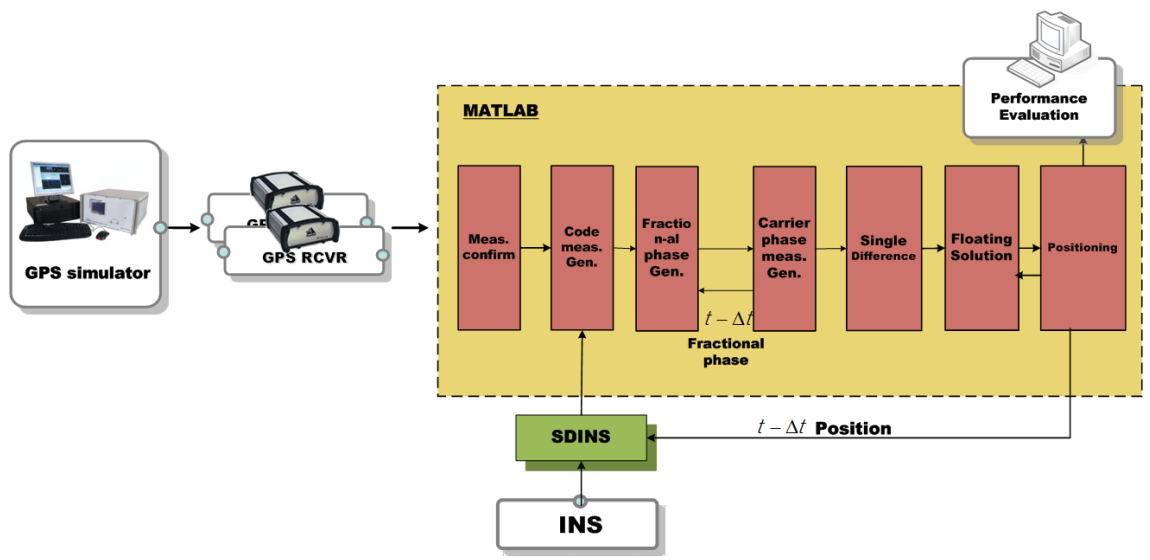

Fig. 7. Configuration of the simulation in a weak or blocked satellite signal environment. 
sections in current epoch $(t)$ even if a satellite signal is weak or blocked. As shown in Fig. 4, the probability for the fractional phase section of current epoch $(t)$ to be the same with the phase sections of previous epoch $(t-\Delta t)$ or located in the previous or next section is totally $87 \%$, and in comparison with actual fractional phase value, it can be expected to generate stochastically accurate fractional phase values using the statistics.

\section{VERIFICATION OF PERFORMANCE}

In order to generate and verify code and carrier phase measurements using the proposed method without any influence on the atmospheric delay errors and other

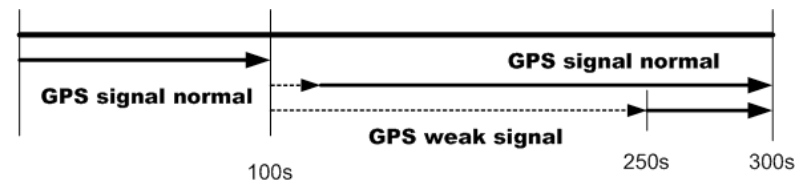

Fig. 8. Configuration for the simulation time. error factors, precise positioning of Single-DGPS (Single Differential GPS) for L1 signal composed of differentials between receivers was used.

The configuration of SDGPS simulation in the static condition is as shown in Fig. 5. There is a difference in the distance within several $\mathrm{km}$ between the receiver in a ship and the receiver in the base station in an actual environment, but the simulation was carried out in zero baseline under the static condition in order not to consider errors due to influence of distance and antennas, and the result is as shown in Fig. 6. It was confirmed through the simulation that the 2 dRMS result showed an estimated position for approximately $17 \mathrm{~cm}$.

The configuration of simulation in a weak or blocked satellite signal environment is as shown in Fig. 7. Code and carrier phase measurements were generated using the proposed algorithm for the blocked satellite signals. GPS simulator and GPS receiver used in Chapter 3 were used to carry out the simulation, and Honeywell's HG1700 which is a tactical inertial sensor is used to maintain the performance of SDGPS navigation. The simulator is used to generate
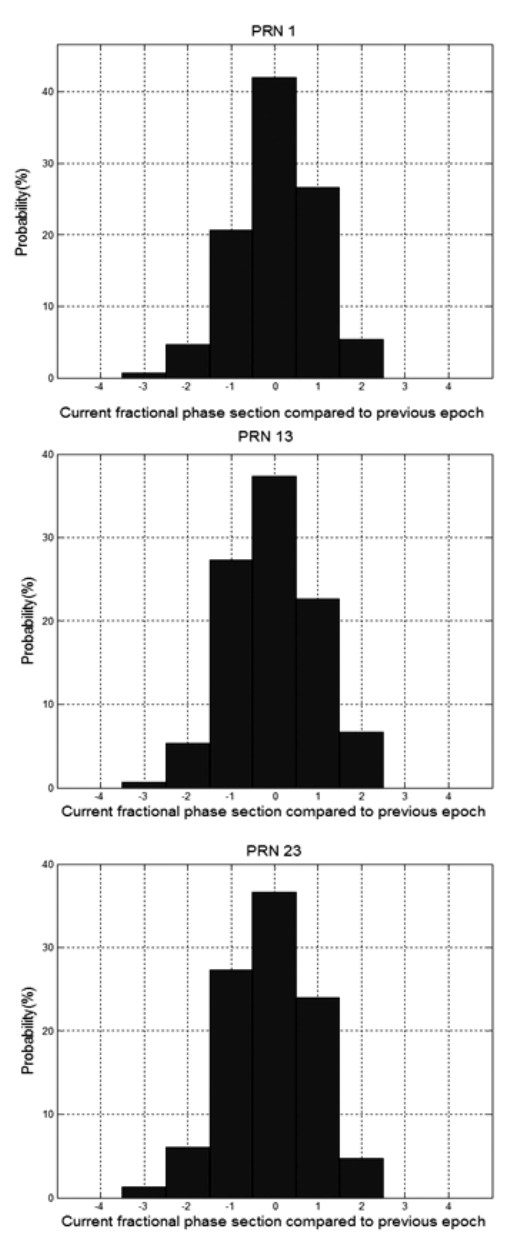

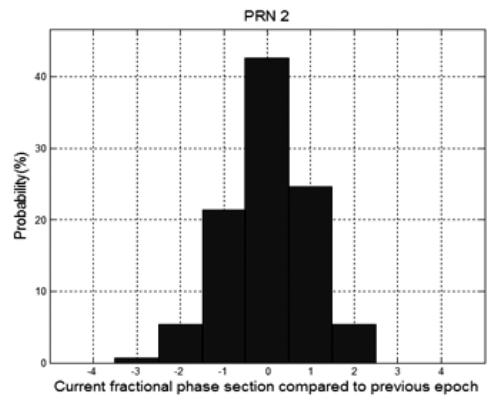

PRN 14
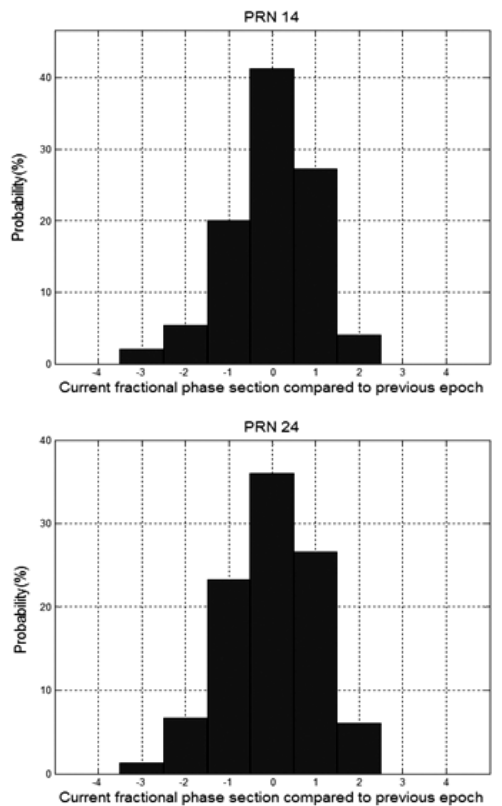
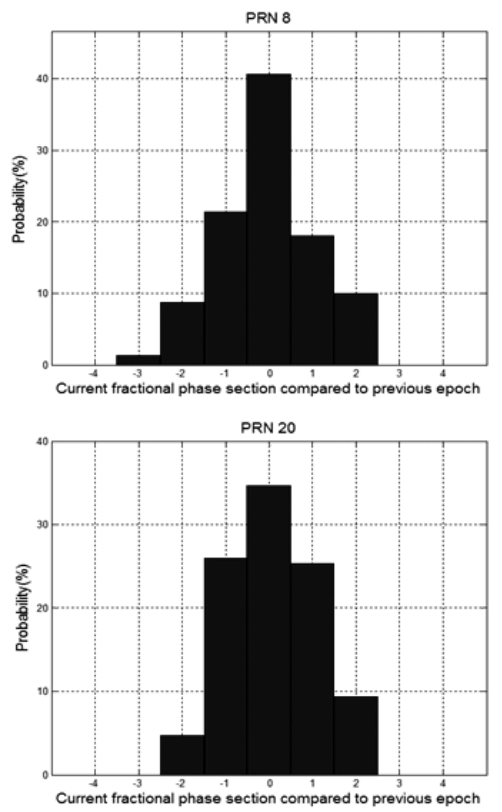

Fig. 9. Fractional phase statistics generated for each satellite when a signal is blocked for 150 seconds. 
Fractional phase statistics for 8 satellite

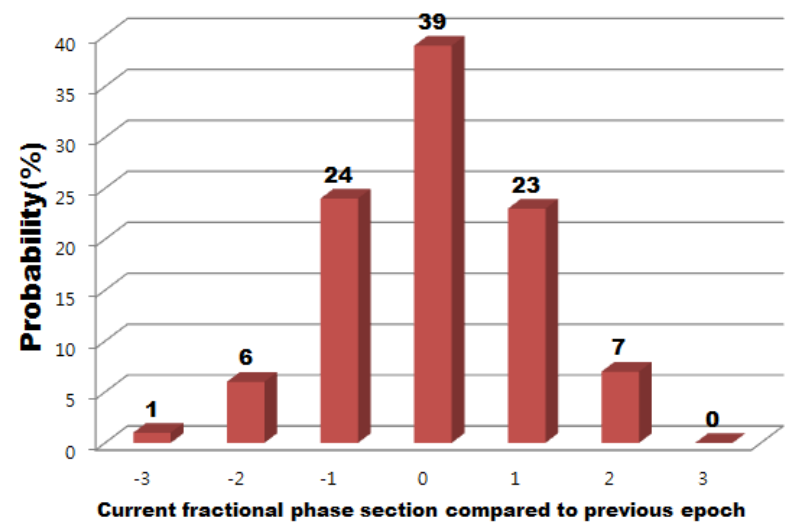

Fig.10. Fractional phase statistics of 8 satellites generated when signals were blocked for 150 seconds.

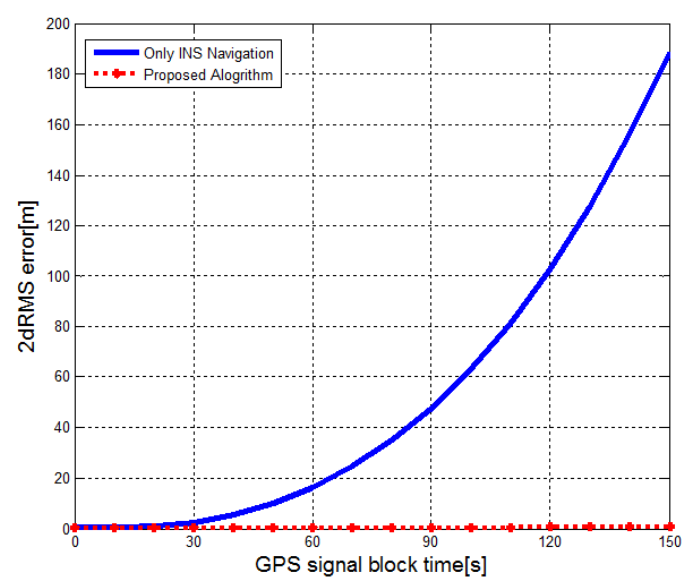

Fig. 11. Comparison of navigation error of two algorithms when a satellite signal is blocked.

satellite signals and the performance was evaluated based on MATLAB.

The duration of simulation was 300 seconds, and the differences in the navigation results according to each time of blocked satellite signals were compared through total 15 simulations in a 10-second interval for 150 seconds with the blocking of all satellite signals. After the simulation began, SDGPS navigation was carried out until 100 seconds in normal condition, and after 100 seconds, all satellite signals were blocked in a 10-second interval until 250 seconds and SDGPS navigation was performed by generating the measurements through the inertial navigation only and the proposed algorithm, and then the SDGPS navigation was performed again in normal satellite signal condition until 300 seconds.

Fig. 9 shows the fractional phase statistics generated for each satellite when all satellite signals are blocked for 150 seconds, and Fig. 10 shows the fractional phase statistics for 8 satellites. The statistics from each satellite shown

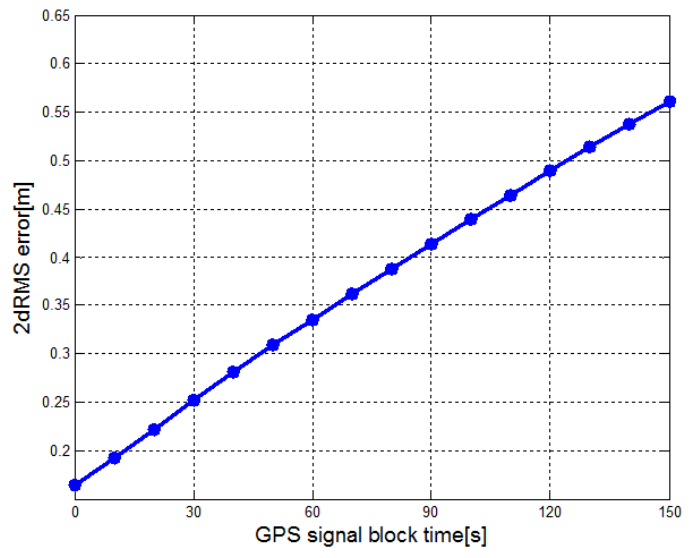

Fig. 12. Navigation error of the proposed algorithm.

in Fig. 9 show a similar pattern with the statistics in Fig. 4 even though there is a probability difference, and the probability of fractional phase statistics of all satellites in Fig. 10 is almost same with the probability of fractional phase statistics in Fig. 4, showing that relatively accurate fractional phase was generated based on the proposed carrier fractional phase statistics.

Fig. 11 shows the result of applying the inertial sensor navigation and the proposed algorithm when all satellite signals are blocked. It shows 2 dRMS error for each blocked time in 10-second interval when blocking the signal from 100 seconds in 10-second interval as shown in Fig. 8, and it was confirmed that the performance in case of applying the proposed algorithm was more improved than the performance in the case of applying the inertial sensor navigation only, and the error increased linearly but its increasing amount is very small. It was also confirmed that in case of a blocked satellite signal for a long period of time, the 2 dRMS result of the inertial navigation only after approximately 1 minute was approximately $17 \mathrm{~m}$, showing a large error, but in case of applying the proposed algorithm, the result was approximately $0.34 \mathrm{~m}$, still satisfying the accuracy for safe navigation or precise positioning. Therefore, resilient positioning accuracy within $1 \mathrm{~m}$ of 2 dRMS can be maintained in case of a blocked satellite signal for 1 to 5 minutes.

\section{SUMMARY AND CONCLUSION}

Previously, navigators determined sailing with approximate predictive values for the air clearance and position of the ship in order to pass through a bridge on the sea or prevent collision with a crossing ship when sailing on the shore or inland waterways. Due to inaccurate predictive values, 
maritime accidents have been occurring consistently. In order to solve this problem, the precise positioning technology using carrier phase information is used, but due to tall buildings or mountain near inland waterways or inclination, satellite signals might be weak or blocked for some time. Under this weak signal environment for some time, the GPS raw measurements become less accurate so that it is difficult to search and maintain carrier phase integer ambiguity and the positioning becomes less accurate.

In this paper, code measurements and carrier phase measurements were generated to maintain the continuity of carrier phase based positioning in a weak or blocked satellite signal environment. In order to verify the proposed method, simulations were carried out, and as a result of confirming the navigation results using the inertial navigation only and the proposed method for each blocked time, the inertial navigation showed error over $10 \mathrm{~m}$ but the proposed method maintained stable accuracy in the positioning with $2 \mathrm{dRMS}$ error within $1 \mathrm{~m}$ in case of a weak or blocked satellite signal for 1 to 5 minutes.

The performance of the proposed algorithm will be reconfirmed through field tests in real-time static and dynamic conditions and the possibility of applying the proposed algorithm in land transportation environment will be considered.

\section{ACKNOWLEDGMENTS}

This work was supported by the Korea Research Council of Fundamental Science \& Technology and Korea Institute of Ocean Science \& Technology for sponsoring this research within the framework of "Development of Satellite based Precise Vertical Positioning Technologies for Safe Maritime Navigation and Early Detection of Disaster Sea Wave".

\section{REFERENCES}

IGS (International GNSS Service). http://igscb.jpl.nasa.gov/ components/prods.html

Kim, J. H., \& Sukkarieh, S. 2003, Autonomous Airborne Navigation in Unknown Terrain Environments, Proceedings of IEEE International Conference on Robotics and Automation, Taipei, pp.13-19

Melgard, T., de Jong, K., Lachapelle, G., \& Lapucha, D. 2011, Interchangeable Integration of GPS and GLONASS by Using a Common System Clock in PPP, Proceedings of ION GNSS 2011, Portland, pp.2179-2184

Niell, A. E. 1996, Global mapping functions for the atmosphere delay at radio wavelengths, JGR, 101(B2), 3227-3246. http://dx.doi.org/10.1029/95JB03048

Parkinson, B. W., \& Spilker, Jr, J. J. 1996, Global Positioning System: Theory and Application, ed. P. Zarchan (Washington D.C.: AIAA)

Saastamoinen, J. 1972, Autonomous correction for the troposphere and stratosphere in radio ranging of satellites, in The Use of Artificial Satellites for Geodesy, Geophys. Monogr. Ser., vol. 15 (AGU: Washington, D.C.)

Teunissen, P. J. G., \& Kleusberg, A. (eds.), 1998, GPS for Geodesy, 2nd Ed (New York: Spreinger-yerlag)

Tiberius, C. \& de Jonge, P. 1995, Introduction to GPS surveying (part 2). GIM International Journal for Geomatics, 9, 71-77

Titterton, D. H., \& Weston, J. L. 2004, Strapdown Inertial Navigation Technology, 2nd Ed (Stevenage: Institution of Electrical Engineers) 